\title{
Effects of Bacterial Cellulose Nanocrystals on the Mechanical Properties of Resin-Modified Glass Ionomer Cements
}

\author{
Marzieh Moradian ${ }^{1}$ Mohsen Nosrat Abadi ${ }^{2}$ Dana Jafarpour ${ }^{3} \quad$ Maryam Saadat $^{1}$ \\ ${ }^{1}$ Department of Operative Dentistry, School of Dentistry, Shiraz \\ Address for correspondence Maryam Saadat, DDS, MSCD, \\ University of Medical Sciences, Shiraz, Iran \\ ${ }^{2}$ Student Research Committee, School of Dentistry, Shiraz \\ University of Medical Sciences, Shiraz, Iran \\ ${ }^{3}$ Biomaterials Research Center, School of Dentistry, Shiraz \\ Department of Operative Dentistry, School of Dentistry, \\ Shiraz University of Medical Sciences, Ghomabad Street, \\ Shiraz 7134518636, Iran \\ (e-mail: dr.maryam.saadat@gmail.com).
}

University of Medical Sciences, Shiraz, Iran

Eur J Dent 2021;15:197-201

\begin{abstract}
Objectives The purpose of this study was to evaluate the effect of bacterial cellulose nanocrystals (BCNCs) on the mechanical properties of resin-modified glass ionomer cements (RMGICs) including compressive strength (CS), diametral tensile strength (DTS), and modulus of elasticity (E).

Materials and Methods BCNCs were incorporated into RMGIC at various concentrations $(0.3,0.5$, and $1 \mathrm{wt} \%)$. Unmodified RMGIC was used as the control group. The specimens were stored in distilled water at $37^{\circ} \mathrm{C}$ for 24 hours. CS and DTS, as well as modulus of elasticity, were evaluated using a universal testing machine. The nanostructure of BCNCs was observed via field emission scanning electron microscopy.

Statistical Analysis One-way analysis of variance and post-hoc Tukey tests were used for data analysis. Level of significance was at $p<0.05$.

Keywords

Results The addition of BCNCs to RMGIC led to an increase in all of the tested

- bacteria mechanical properties compared with the control group, with a significant increase

- cellulose observed for $1 \mathrm{wt} \%$ BCNC. CS and DTS improved up to $23 \%$, and modulus of elasticity

- compressive strength

- elastic modulus increased by $44 \%$.

- glass ionomer Conclusions The addition of BCNCs to the RMGIC improved the mechanical properties, including CS, elastic modulus, and DTS. Thus, the newly developed RMGICs with cements

- tensile strength BCNCs might represent an ideal and promising novel dental material in restorative dentistry.
\end{abstract}

\section{Introduction}

Glass ionomer cements (GICs), which were introduced by Wilson and Kent in the early 1970s, are considered as the treatment choice in various clinical situations since they offer great benefits. These advantages include chemical adhesion to tooth structure, anticariogenic activity due to fluoride release, biocompatibility, and low coefficient of thermal expansion, which makes it similar to that of tooth structure. ${ }^{1}$ However, some drawbacks such as low mechanical strength, brittleness, and low wear resistance have made GICs far from being used as direct restorative materials in the load-bearing area. ${ }^{2}$
DOI https://doi.org/

10.1055/s-0040-1717051

ISSN 1305-7456. (c) 2020. European Journal of Dentistry.

This is an open access article published by Thieme under the terms of the Creative Commons Attribution-NonDerivative-NonCommercial-License, permitting copying and reproduction so long as the original work is given appropriate credit. Contents may not be used for commercial purposes, or adapted, remixed, transformed or built upon. (https://creativecommons.org/licenses/by-nc-nd/4.0/) Thieme Medical and Scientific Publishers Pvt. Ltd., A-12, 2nd Floor, Sector 2, Noida-201301 UP, India 
To fortify the benefits and overcome the shortcomings of the conventional GIC, resin-modified glass ionomer cements (RMGICs) were introduced. Yet, the addition of 2-hydroxyethyl methacrylate (HEMA) in RMGICs led to greater water uptake and swelling of the resin matrix due to the hydrophilic composition of HEMA. ${ }^{3}$ Water leads to hydrolysis and plasticization of the resin-polyacrylate matrix, which might in turn deteriorate the mechanical properties of the cement. ${ }^{4}$ Therefore, RMGICs possess some desired properties over their conventional counterparts. However, drawbacks of RMGICs still need to be overcome. These deficits principally origin from their weak mechanical strength, including wear resistance, compressive strength (CS), and diametral tensile strength (DTS).

Nowadays, novel GIC designs have been introduced and various methods have been described by several researchers to ameliorate the mechanical and physical properties of glass ionomer restoratives. For instance, the addition of amalgam into the GIC powder was performed with the aim of refining the mechanical properties of these restorative materials. Yet, this combination has led to a diminished esthetics and lower bond strength to the enamel. ${ }^{5}$ The mechanical properties of GICs have also been improved by integrating with short silicon carbide fibers. ${ }^{6}$ However, it has been reported that these tiny fibers can accumulate in vital organs and produce toxicity similar to those of asbestos fibers. ${ }^{7}$ Moreover, numerous efforts have been conducted to enhance the GIC fillers by means of adding filler particles including hydroxyapatite, ${ }^{8}$ zirconia, ${ }^{9}$ ytterbium fluoride/ barium sulfate, ${ }^{10}$ and silver ${ }^{11}$ into GICs. Yet, none of these methods have led to considerable reinforcements in wear resistance and mechanical strengths of GICs.

The great advancement in nanotechnology along with an urge for viable developments have led to the popularity of cellulose application because of its sustainable properties, harmlessness, abundance, low density, and little thermal expansion. ${ }^{12}$ The cellulose nanocrystals (CNCs) are extremely crystalline cellulose-derived structures that demonstrate outstanding mechanical strength. The CNCs, which have an average of 100 to $250 \mathrm{~nm}$ length and 5 to 15 $\mathrm{nm}$ diameter, are formed by acid hydrolysis of any natural source of cellulose. Nowadays, cellulose nanoparticles are among the most widely used materials in the improvement of mechanical properties in dental practice. CNCs form a solid scaffold in different directions within the material and greatly improve the mechanical properties by establishing hydrogen bonds. ${ }^{13,14}$ It is noteworthy to mention that the cellulose produced by some bacteria has a nanometer-sized width, even before going through processing. Bacterial cellulose is unique due to its high crystallinity, ${ }^{15}$ high water retention capability, ${ }^{16}$ and excellent mechanical ${ }^{17}$ and thermal properties, ${ }^{18}$ which is why many researchers prefer bacterial nanocelluloses for medical applications.

Although several studies have been performed to investigate various mechanical properties of RMGICs, ${ }^{19-22}$ no research has been conducted to assess the effect of bacterial cellulose nanocrystals (BCNCs) on mechanical properties of RMGICs. Therefore, the purpose of this study was to evaluate the effect of BCNCs in three volumetric masses of $0.3,0.5$, and $1 \mathrm{wt} \%$ on the mechanical properties of RMGICs. The null hypothesis of our study was that there would be no difference between the mechanical properties of RMGIC and BCNC-containing RMGIC.

\section{Materials and Methods}

A total of 80 specimens were prepared in this study. The specimens were divided into four main groups $(n=20)$ : Group I (RMGIC powder, control), Group II (RMGIC powder with $0.3 \mathrm{wt} \% \mathrm{BCNC}$ ), Group III (RMGIC powder with $0.5 \mathrm{wt} \%$ BCNC), and Group IV (RMGIC powder with $1 \mathrm{wt} \% \mathrm{BCNC}$ ). Then the specimens of each group were divided into two subgroups for the CS and DTS tests $(n=10)$.

\section{Sample Preparation}

In this study, BCNC powder (Nano Novin Polymer Co.; Gorgan, Golestan, Iran) was used. Bacterial cellulose was extracted from Gluconacetobacter genus.

BCNC powders were weighed carefully to an accuracy of $0.001 \mathrm{~g}$ by means of a digital scale (GR-3000, A \& D CL Toshiba, Tokyo, Japan) and were added to the previously weighed RMGIC powder (Fuji II, GC, Tokyo, Japan) containing 95\% fluoroaluminosilicate glass (amorphous) and 5\% polyacrylic acid using the correct concentration for each group $(0.3$, 0.5 , or $1 \mathrm{wt} \%$ ). To obtain a uniform powder in the specimens, initially RMGIC and BCNC powders were hand mixed and then the obtained powder was placed in amalgam capsules in an amalgamator (Ultramat 2, SDI, Australia) for 20 seconds. ${ }^{23}$

Then the resultant powder was mixed with RMGIC liquid (Fuji II) containing 20 to 30\% distilled water, 20 to 30\% polyacrylic acid, and 30 to $35 \%$ HEMA $^{24}$ in accordance with manufacturer's instructions (3/2 g: $1 \mathrm{~g}$ ).

To determine CS and DTS, specimens were prepared in a cylindrical stainless-steel split mold (4 mm diameter and $8 \mathrm{~mm}$ height) in the same procedure described by ISO 9917-1:2017.25

The mold was placed on the top of Mylar strip rested on a glass plate and the mold was filled with the material. Then a second piece of Mylar strip was placed on the material in the mold and pressed by another glass plate under hand pressure to remove excess material. The light cured specimens were eradicated for 20 seconds trough Mylar strip using light-emitting diode at light intensity of $1,500 \mathrm{~mW} / \mathrm{cm}^{2}$ and a wavelength range of 440 to $480 \mathrm{~nm}$ (Coltolux II, Coltene, Ohio, United States). The glass plate and celluloid Mylar strip were then carefully removed. After removing the specimens, the specimens were cured from the other side to ensure that the samples were completely cured.

The specimens were placed in distilled water $\left(37^{\circ} \mathrm{C}\right)$ for 24 hours prior to the experiment. For each test (CS and DTS), a total of 40 samples ( $n=10$ ) were placed in the universal testing machine (Instron, Z020. Zwick Roell, Germany).

\section{Compressive Strength Test}

For the CS measurement, the specimens were located lengthwise between the platens of the machine and compressed at a crosshead speed of $1 \mathrm{~mm} / \mathrm{min}$. The maximum load required to fracture each specimen was documented 
and the CS in mega Pascal (MPa) was measured using this formula: CS: $4 F / \pi d 2$

Where $d$ is sample diameter and $F$ is the force loaded at the moment of fracture.

\section{Diametral Tensile Strength Test}

For the DTS, the samples were placed between the platens of the machine along their diameter. The tensile strength was measured according to the following formula:

Diametral traction tension: $2 F / \pi d l$

Where $d$ is sample diameter, $F$ is the force loaded at the moment of fracture, and $l$ is the primary sample length.

\section{Modulus of Elasticity}

Young's modulus for each specimen was determined according to the slope of the plotted graphs for CS produced by the universal testing machine.

$$
E: \sigma / \varepsilon=(F / A) /(\Delta L / L)
$$

Where $A$ is the diagonal cross-section, $\Delta L$ is the length change during compression test, $L$ is the primary sample length, and $F$ is the force loaded at the moment of fracture.

\section{Field Emission Scanning Electron Microscopy}

For field emission scanning electron microscopy (FESEM) analysis, a thin layer of gold-palladium was used to cover the representative specimen in BCNC experimental group. Using 10-15 kV electron beam, the specimens were witnessed by FESEM (CS-3500, Shimadzu, Kyoto, Japan) at magnifications of $1,000 \times$ and 75,000× ( - Fig. 1).

\section{Statistical Analysis}

For data analysis, the mean and standard deviation (SD) values of all groups were obtained using Statistical Package for the Social Sciences version 15.0 (Microsoft, Illinois, United States). The average and SD were used for data description. To evaluate the homogeneity of data, the Kolmogorov-Smirnov test was used. One-way analysis of variance (ANOVA) and post-hoc Tukey tests were used for data analysis. Significance level was $p<0.05$.
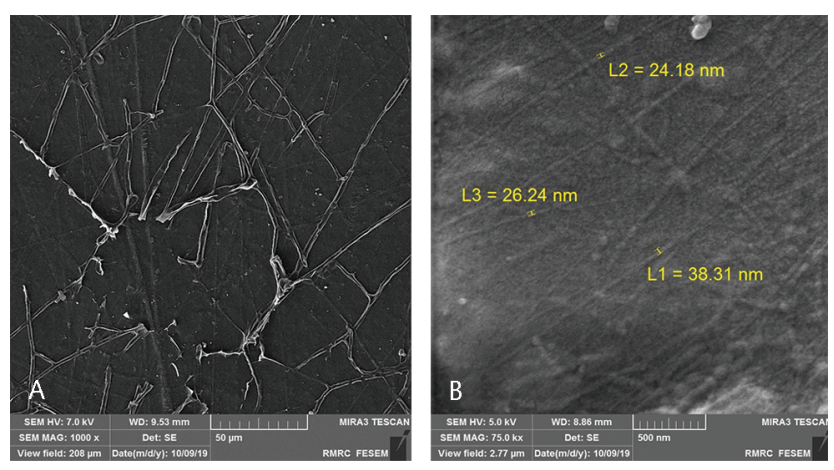

Fig. 1 Field emission scanning electron microscopy images of 1 wt\% bacterial cellulose nanocrystal BCNC-containing resin-modified glass ionomer cement: (A) 1,000× magnification showing the net-like structure of BCNC within cementitious mass; (B) 75,000× magnification showing the size of BCNC.

\section{Results}

The mean \pm SD values of CS, DTS, and modulus of elasticity $(E)$ of various trial groups are shown in - Table 1. The results of one-way ANOVA revealed that there was a significant difference between CS values of different tested groups $(p=0.010)$.

As shown in - Table 1, the highest CS was observed in group IV ( $1 \% \mathrm{wt})$, which was significantly greater than the CS of the control group ( $p=0.007)$. No significant difference was found between the CS of the $1 \% \mathrm{wt}$ group and that of $0.5 \% \mathrm{wt}$ $(p=0.804)$ and $0.3 \% \mathrm{w} / \mathrm{w}(p=0.565)$.

Furthermore, a significant difference was observed between modulus of elasticity $(E)$ of the CS values of different experimental groups $(p<0.001)$.

As shown in - Table 1, the modulus of elasticity $(E)$ of the CS values of the control group was significantly lower than $0.3 \% \mathrm{w} / \mathrm{w}, 0.5 \% \mathrm{wt}$, and $1 \% \mathrm{wt}$ groups $(p<0.001)$.

Moreover, there was a significant difference between DTS values of different experimental groups $(p=0.002)$.

As revealed in - Table 1, the highest DTS was observed in group IV (1\% wt), which was significantly greater than the DTS of the control group $(p=0.001)$. No significant difference was found between the DTS of the $1 \mathrm{wt}$ group and that of $0.5 \%$ wt $(p=0.249)$ and $0.3 \%$ wt $(p=0.055)$.

-Figure 1 shows representative FESEM images of $1 \mathrm{wt} \%$ BCNC in cementitious mass of RMGIC with a net-like structure and reveals the size of BCNC.

\section{Discussion}

The current study evaluated the effect of adding BCNCs on the mechanical properties of RMGICs. The null hypothesis was rejected. The result of this study showed that the RMGICs containing $1 \mathrm{wt} \%$ BCNCs represented significantly higher CS and DTS compared with the control group. Thus, adding BCNC to the RMGIC can increase the strength of the newly developed GICs without any difficulty with regard to clinical applications.

Nowadays, attention has been given to modern technologies in dentistry for the introduction of new restorative materials. In this regard, the introduction of cellulosic fibers, especially CNCs, aimed to meet such demands and were in line with the objectives for the production of renewable biomaterials and green development. ${ }^{26}$

The cellulose examined in the present study was derived from bacteria as this type of cellulose can be readily processed into nanocrystals. These nanocrystals are applied as strengthening constituents in producing polymers with high-performance applications. Bacterial cellulose presents a wider range of application compared with its herbal counterparts due to its exclusive thermo-mechanical characteristics and biocompatibility. ${ }^{27,28}$ Therefore, the reinforced mechanical properties of bacterial cellulose-modified GICs might offer more durable and long-term restorations prepared with this restorative cement.

Previously, low concentrations of CNCs have been found considerably sufficient to enhance the mechanical strength of composites. In fact, according to Silva et $\mathrm{al}^{29}$ when the 
Table 1 Mean \pm standard deviation values of compressive strength, diametral tensile strength, and modulus of elasticity of various experimental groups

\begin{tabular}{|l|l|l|l|}
\hline Study group & Compressive strength & Diametral tensile strength & Modulus of elasticity \\
\hline G I: Control & $133.60 \pm 17.35^{\mathrm{b}}$ & $18.64 \pm 2.53^{\mathrm{b}}$ & $1,151.60 \pm 163.56^{\mathrm{b}}$ \\
\hline G II: $0.3 \mathrm{wt} \%$ & $152.61 \pm 23.97^{\mathrm{ab}}$ & $20.16 \pm 2.52^{\mathrm{ab}}$ & $1,674.30 \pm 164.95^{\mathrm{a}}$ \\
\hline G III: $0.5 \mathrm{wt} \%$ & $156.73 \pm 17.01^{\mathrm{ab}}$ & $20.92 \pm 2.61^{\mathrm{ab}}$ & $1,683.48 \pm 193.89^{\mathrm{a}}$ \\
\hline G IV: $1 \mathrm{wt} \%$ & $164.54 \pm 22.55^{\mathrm{a}}$ & $22.84 \pm 2.32^{\mathrm{a}}$ & $1,663.64 \pm 226.27^{\mathrm{a}}$ \\
\hline
\end{tabular}

Note: Different lower-case letters (superscripts) indicate significant differences between different groups $(p<0.05)$.

concentrations of CNCs were more than $1 \mathrm{wt} \%$, the nanoparticles accumulated, leading to the failure of composites' mechanical properties. Thus, in the present study, concentrations of $0.3,0.5$, and $1 \mathrm{wt} \%$ of BCNCs were used for the investigation of mechanical properties.

The results showed that the addition of $1 \mathrm{wt} \% \mathrm{BCNC}$ to RMGIC led to a significant increase in the mechanical properties of the RMGIC. Furthermore, the addition of $0.3,0.5$, and $1 \mathrm{wt} \%$ of BCNCs to RMGICs resulted in a significant increase in the modulus of elasticity of the CS of these materials compared with the control group.

CS and flexural strength are regarded as load-bearing capacity indicators of a restorative dental material..$^{30} \mathrm{GIC}$ is a brittle material with a tensile strength distinctly lower than its CS. In fact, this material fails by dissemination of crack under tensile rather than compressive forces. ${ }^{31}$ Studying scanning electron microscopy confirmed that the addition of nanoparticles reduced porosity, with no differences between the materials. ${ }^{22}$ Therefore, it seems that the addition of BCNCs can fill spaces in the defects and impede the dissemination of previously present pores by blocking their paths, which in turn stops the pores from forming cracks and improves the DTS of RMGICs. It has been shown that an improvement in this property is hardly achievable in GICs. ${ }^{32}$ Therefore, this finding can be of high clinical significance since it is essential for GICs located in the load-bearing areas to resist the masticatory occlusal forces produced within their structure.

The FESEM image in this study revealed that the incorporation of BCNC to the RMGIC led to the creation of an interconnected network of BCNC, which was randomly distributed within the GIC matrix. Silva et al ${ }^{29}$ have previously shown that the nanoscale size of the $\mathrm{CNC}$ fibers assists the dissemination of CNCs in the GIC matrix and facilitates the creation of a weblike arrangement within the matrix. As a consequence, this significant improvement in the mechanical properties of the BCNC-incorporated RMGICs can be attributed to the uniform dissemination of the interrelated nanocrystals in the cement matrix. In addition, BCNC's ability to form a hydrogen bonding with the hydroxyl groups of the glass particles and carboxylic groups of the polyacrylic acid can be another possible explanation for the RMGIC's improved mechanical strength.

The formation of the network made by BCNCs adhering to the GIC particles may be explicated by the inherent self-association of the BCNCs. In fact, an electrostatic interaction occurs between the positive charges of the GIC and the negative charges of the BCNC that, in turn, causes cement reinforcement. ${ }^{33}$ This innate feature of the BCNC also helps in forming auxiliary architectures for the percolation of load into the cement matrix. ${ }^{34}$ Following this network formation, a substantial strengthening effect of the RMGIC was found in all the tested properties. DTS, CS, and elastic modulus increased from 18.64, 133.60, and 1,151.60 MPa in the control group to $22.84,164.54$, and $1,663.64 \mathrm{MPa}$ in the $1 \mathrm{wt} \%$ added BCNCs, respectively.

In line with our findings, Silva et a ${ }^{29,35,36}$ showed that the addition of a small amount of a nanoparticulate renewable material in the form of CNCs significantly increased the CS and DTS strengths of restorative GICs. In a recent study, CNCs were used in combination with titanium oxide $\left(\mathrm{TiO}_{2}\right)$ nanoparticles as an additive to GIC. It was reported that the physical properties of the modified GIC reinforced with $2 \mathrm{wt} \% \mathrm{TiO}_{2}$ nanoparticles and $1 \mathrm{wt} \%$ of $\mathrm{CNC}$ showed significant improvement. ${ }^{37}$

Given the desired mechanical strength of the BCNCincorporated RMGICs observed in this study, the RMGIC modified with $1 \mathrm{wt} \%$ BCNC might provide a practicable restorative material to be used under stress, which could help decrease tooth loss.

One of the limitations of this study was that the current research was conducted in-vitro. The in-vitro results obtained in the present study do not necessarily validate those achieved in vivo. For this matter, there is an urge for clinical studies to confirm in-vitro findings. Moreover, only compressive and tensile tests were performed in our study. The future of RMGICs modified with BCNCs still requires further investigations on other properties of these novel materials. Properties such as color stability and biocompatibility are needed to be studied for the comprehensive finding of the properties and characteristics of these materials.

\section{Conclusions}

It was found that the addition of $1 \mathrm{wt} \%$ of BCNC to the RMGIC considerably improved all the tested properties, including CS, DTS, and elastic modulus. Thus, the newly developed RMGICs with BCNCs might represent an ideal and promising novel dental material in restorative dentistry.

\section{Conflict of Interest}

None declared.

\section{Acknowledgments}

The authors thank the vice-chancellery of Shiraz University of Medical Science for supporting this research (Grant \#18732). This manuscript is based on the 
thesis by Mohsen Nosrat Abadi. The authors also thank Dr. Vossoughi of the Center for Research Improvement of the School of Dentistry for the statistical analysis.

\section{References}

1 Wilson AD \& McLean JW. $1^{\text {st }}$ ed. Glass-ionomer cement. Chicago: Quintessence Pub Co; 1988:43-56

2 Ilie N, Hickel R, Valceanu AS, Huth KC. Fracture toughness of dental restorative materials. Clin Oral Investig 2012;16(2):489-498

3 Kanchanavasita W, Anstice HM, Pearson GJ. Water sorption characteristics of resin-modified glass-ionomer cements. Biomaterials 1997;18(4):343-349

4 Kanchanavasita W, Anstice HM, Pearson GJ. Long-term surface micro-hardness of resin-modified glass ionomers. J Dent 1998;26(8):707-712

5 Irie M, Nakai H. Mechanical properties of silver-added glass ionomers and their bond strength to human tooth. Dent Mater J 1988;7(1):87-93

6 Kobayashi M, Kon M, Miyai K, Asaoka K. Strengthening of glass-ionomer cement by compounding short fibres with CaO-P2O5-SiO2-Al2O3 glass. Biomaterials 2000;21(20): 2051-2058

7 Arita K, Yamamoto A, Shinonaga Y, et al. Hydroxyapatite particle characteristics influence the enhancement of the mechanical and chemical properties of conventional restorative glass ionomer cement. Dent Mater J 2011;30(5):672-683

8 Yap AU, Pek YS, Kumar RA, Cheang P, Khor KA. Experimental studies on a new bioactive material: HAlonomer cements. Biomaterials 2002;23(3):955-962

9 Gu YW, Yap AUJ, Cheang P, Koh YL, Khor KA. Development of zirconia-glass ionomer cement composites. J Non-Cryst Solids 2005;351:508-514

10 Prentice LH, Tyas MJ, Burrow MF. The effect of ytterbium fluoride and barium sulphate nanoparticles on the reactivity and strength of a glass-ionomer cement. Dent Mater 2006;22(8):746-751

$11 \mathrm{Gu} \mathrm{B}$, Sen A. Synthesis of aluminum oxide/gradient copolymer composites by atom transfer radical polymerization. Macromolecules 2002;35:8913-8916

12 Azizi Samir MAS, Alloin F, Dufresne A. Review of recent research into cellulosic whiskers, their properties and their application in nanocomposite field. Biomacromolecules 2005;6(2):612-626

13 GeorgeJ, Ramana KV, Bawa AS. Siddaramaiah. Bacterial cellulose nanocrystals exhibiting high thermal stability and their polymer nanocomposites. Int J Biol Macromol 2011;48(1):50-57

14 Jasmani L, Thielemans W. Preparation of nanocellulose and its potential application. For Res 2018;7:222-228

15 Hult E-L, Yamanaka S, Ishihara M, Sugiyama J. Aggregation of ribbons in bacterial cellulose induced by high pressure incubation. Carbohydr Polym 2003;53:9-14

16 Bäckdahl H, Helenius G, Bodin A, et al. Mechanical properties of bacterial cellulose and interactions with smooth muscle cells. Biomaterials 2006;27(9):2141-2149

17 Iguchi M, Yamanaka S, Budhiono A. Bacterial cellulose-a masterpiece of nature's arts. J Mater Sci 2000;35:261-270

18 George J, Sajeevkumar VA, Kumar R, Ramana KV, Sabapathy SN, Bawa AS. Enhancement of thermal stability associated with the chemical treatment of bacterial (Gluconacetobacter xylinus) cellulose. J Appl Polym Sci 2008;108:1845-1851

19 Karimi M, Hesaraki S, Alizadeh M, Kazemzadeh A. Effect of synthetic amorphous calcium phosphate nanoparticles on the physicochemical and biological properties of resin-modified glass ionomer cements. Mater Sci Eng C 2019;98:227-240

20 Felemban NH, Ebrahim MI. Effects of adding silica particles on certain properties of resin-modified glass-ionomer cement. Eur J Dent 2016;10(2):225-229

21 Moheet IA, Luddin N, Rahman IA. Kannan TP, Nik Abd Ghani NR, Masudi SM. Modifications of glass ionomer cement powder by addition of recently fabricated nano-fillers and their effect on the properties: a review. Eur J Dent 2019;13(3):470-477

22 Nicholson JW, Sidhu SK, Czarnecka B. Enhancing the mechanical properties of glass-ionomer dental cements: a review. Materials (Basel) 2020;13(11):2510-2523

23 Sharafeddin F, Moradian M, Motamedi M. Evaluation of shear bond strength of methacrylate-and silorane-based composite resin bonded to resin-modified glass-ionomer containing micro-and nano-hydroxyapatite. J Dent (Shiraz) 2016;17(2):142-148

24 Omidi BR, Naeini FF, Dehghan H, Tamiz P, Savadroodbari MM, Jabbarian R. Microleakage of an enhanced resin-modified glass ionomer restorative material in primary molars. J Dent (Tehran) 2018;15(4):205-213

25 International Organization for Standardization (ISO). Dentistry-Water-Based Cements-Part 1: Powder/Liquid AcidBase Cements. ISO 9917-1:2017. Geneva, Switzerland, 2017

26 Mariano M, El Kissi N, Dufresne A. Cellulose nanocrystals and related nanocomposites: review of some properties and challenges. J Polym Sci, B, Polym Phys 2014;52:791-806

27 Ifuku S, Tsuji M, Morimoto M, Saimoto H, Yano H. Synthesis of silver nanoparticles templated by TEMPO-mediated oxidized bacterial cellulose nanofibers. Biomacromolecules 2009;10(9):2714-2717

28 Singhsa P, Narain R, Manuspiya H. Bacterial cellulose nanocrystals (BCNC) preparation and characterization from three bacterial cellulose sources and development of functionalized BCNCs as nucleic acid delivery systems. ACS Appl Nano Mater 2017;1:209-221

29 Silva RM, Pereira FV, Mota FAP, Watanabe E, Soares SMC, Santos MH. Dental glass ionomer cement reinforced by cellulose microfibers and cellulose nanocrystals. Mater Sci Eng C 2016;58:389-395

30 Jowkar Z, Jowkar M, Shafiei F. Mechanical and dentin bond strength properties of the nanosilver enriched glass ionomer cement. J Clin Exp Dent 2019;11(3):e275-e281

31 Garoushi S, Vallittu P, Lassila L. Hollow glass fibers in reinforcing glass ionomer cements. Dent Mater 2017;33(2):e86-e93

32 Yamazaki T, Schricker SR, Brantley WA, Culbertson BM, Johnston W. Viscoelastic behavior and fracture toughness of six glass-ionomer cements. J Prosthet Dent 2006;96(4):266-272

33 de Mesquita JP, Donnici CL, Pereira FV. Biobased nanocomposites from layer-by-layer assembly of cellulose nanowhiskers with chitosan. Biomacromolecules 2010;11(2):473-480

34 Capadona JR, Shanmuganathan K, Tyler DJ, Rowan SJ, Weder C. Stimuli-responsive polymer nanocomposites inspired by the sea cucumber dermis. Science 2008;319(5868) :1370-1374

35 Menezes-Silva R, de Oliveira BM, Fernandes PHM, et al. Effects of the reinforced cellulose nanocrystals on glass-ionomer cements. Dent Mater 2019;35(4):564-573

36 Silva RM, Santos PHN, Souza LB, Dumont VC, Soares JA, Santos MH. Effects of cellulose fibers on the physical and chemical properties of glass ionomer dental restorative materials. Mater Res Bull 2013;48:118-126

37 Sun J, Xu Y, Zhu B, et al. Synergistic effects of titanium dioxide and cellulose on the properties of glass ionomer cement. Dent Mater J 2019;38(1):41-51 